\title{
Associação entre osteoartrite de joelho e síndrome metabólica em pacientes idosos não institucionalizados*
}

\section{Association between Knee Osteoarthritis and Metabolic Syndrome in Non-Institutionalized Elderly Patients}

\author{
Maura Fernandes Franco ${ }^{10}$ Glaucia Regina Falsarella ${ }^{20}$ Beatriz Lavras Costallat ${ }^{3}$ \\ Ibsen Bellini Coimbra ${ }^{2} \odot$ Arlete Maria Valente Coimbra ${ }^{2} \odot$ \\ ${ }^{1}$ Programa de Gerontologia, Faculdade de Ciências Médicas, \\ Universidade Estadual de Campinas, Campinas, SP, Brasil \\ 2 Faculdade de Ciências Médicas, Universidade Estadual de Campinas, \\ Campinas, SP, Brasil \\ ${ }^{3}$ Departamento de Radiologia, Hospital Vera Cruz, Campinas, Brasil \\ Endereço para correspondência Maura Fernandes Franco, Faculdade \\ de Ciências Médicas, Universidade Estadual de Campinas, Rua Tessália \\ Vieira de Camargo, 126 - Cidade Universitária Zeferino Vaz, Campinas, \\ SP 13083-887, Brasil (e-mail: mauraffranco@gmail.com).
} Rev Bras Ortop 2020;55(3):310-316.

\section{Resumo \\ Palavras-chave \\ - osteoartrite \\ - síndrome metabólica \\ - obesidade \\ - envelhecimento \\ - promoção da saúde}

\section{Abstract}

Objetivo Este estudo teve o objetivo de analisar a associação entre a osteoartrite (OA) de joelho e a síndrome metabólica (SM) em pacientes idosos não institucionalizados. Métodos Pesquisa transversal, aleatorizada, extraída de um estudo probabilístico por conglomerado realizado com 416 idosos de uma Unidade de Saúde da Família do nosso município. A SM foi definida de acordo com o National Cholesterol Education Program Adult Treatment Panel III (NCEP-ATP III), e a OA de acordo com a escala Kellgren-Lawrence (KL) $(\geq 2)$.

Resultados Para a análise estatística, foi realizada uma análise exploratória de dados, testes de Mann-Whitney ou Qui-quadrado e regressões logísticas uni e multivariadas, com nível de significância de $p<0,05$; a concordância entre os avaliadores foi verificada através do coeficiente de Kappa. Verificou-se associação entre OA e índice de massa corpórea (IMC) $(p=0,0021)$ e entre OA e circunferência de cintura (CC) $(p<0,001$; razão de chances $[R C]=3,524)$. Não foi encontrada associação significativa entre a $O A$ e o número de componentes metabólicos nem com a SM em si.

Conclusão Conclui-se que a OA de joelho associa-se à CC, independente do peso, e que o aumento em sua medida reflete em uma maior chance de SM em idosos não institucionalizados.

Objective This study aimed to analyze the association between knee osteoarthritis (OA) and metabolic syndrome (MS) in non-institutionalized elderly patients.

Methods A cross-sectional, randomized study, drawn from a probabilistic cluster study conducted with 416 elderly people from a Family Health Unit (USF, in the Portuguese acronym) of our municipality. Metabolic syndrome was defined according

\footnotetext{
Trabalho desenvolvido na Faculdade de Ciências Médicas, Universidade Estadual de Campinas, Campinas, SP, Brasil.
}

recebido

10 de Outubro de 2018 aceito

19 de Março de 2019
DOI https://doi.org/ 10.1055/s-0040-1701281. ISSN 0102-3616.
Copyright $\odot 2020$ by Sociedade Brasileira License terms de Ortopedia e Traumatologia. Published by Thieme Revinter Publicações Ltda, Rio de Janeiro, Brazil 


\section{Keywords}

- osteoarthritis

- metabolic syndrome

- obesity

- aging

- health promotion to the National Cholesterol Education Program Adult Treatment Panel III (NCEP-ATP III), and $\mathrm{OA}$ according to the Kellgren-Lawrence (KL) scale ( $\geq 2$ ).

Results For the statistical analysis, we performed an exploratory data analysis, MannWhitney or Chi-Squared tests and univariate and multivariate logistic regressions, with significance level of $p<0.05$; the concordance between the evaluators was verified through the Kappa coefficient. There was an association between OA and body mass index (BMI) $(p=0.0021)$ and between OA and waist circumference (WC) $(p<0.001$; odds ratio $[O R]=3.524)$. There was no significant association between $O A$ and the number of metabolic components nor with SM itself.

Conclusion We conclude that knee OA is associated with WC, regardless of weight, and that the increase in its measure reflects a greater chance of MS in non-institutionalized elderly patients.

\section{Introdução}

O aumento da ingestão de gordura e do sedentarismo está em evidência no mundo moderno. ${ }^{1}$ Ambos têm influência direta na crescente prevalência de obesidade, dislipidemia, hipercolesterolemia e de uma condição clínica atualmente denominada de síndrome de resistência à insulina ou "síndrome metabólica" (SM). ${ }^{2}$

O estudo da SM tem sido dificultado pela ausência de um consenso em sua definição e nos pontos de corte de seus componentes. A Organização Mundial da Saúde (OMS) ${ }^{3}$ sugeriu uma definição baseada em dados clínicos e laboratoriais com ponto de partida na avaliação da resistência à insulina ou do distúrbio do metabolismo da glicose. Em 2001, o National Cholesterol Education Program's Adult Treatment Panel III (NCEP-ATP III) ${ }^{4}$ propôs uma série de critérios semelhantes, porém mais simples de serem avaliados, os quais incluem: glicemia de jejum, pressão arterial sistólica (PAS), circunferência de cintura (CC), triglicerídeos e highdensity lipoprotein (HDL), facilitando o uso clínico. Ainda surgiram outras definições, tais como as da American Association of Clinical Endocrinologists (AACE) ${ }^{5}$ e do Europoean Group for the Study of Insulin Resistance (EGIR), ${ }^{6}$ mas a da OMS e a do NCEP são as mais utilizadas.

A síndrome metabólica é um transtorno complexo representado por um conjunto de fatores de risco cardiovascular, normalmente relacionados à deposição central de gordura e à resistência à insulina, e deve ser destacada a sua importância do ponto de vista epidemiológico, já que é responsável pelo aumento da mortalidade cardiovascular estimada em 2,5 vezes. ${ }^{7}$ Além disso, as pesquisas recentes têm apontado que essas alterações metabólicas talvez também possam influenciar o aumento da incidência e da progressão da OA., ${ }^{2,8,9}$

Neste sentido, a literatura destaca que os possíveis mecanismos patogênicos em comum entre as doenças osteoarticulares e as doenças metabólicas são a inflamação de baixo grau relacionada ao tecido adiposo e o estresse oxidativo. ${ }^{10-13}$ Alguns pesquisadores apontam que esses mecanismos parecem ter efeitos sistêmicos diretos sobre a articulação e poderiam danificar a cartilagem, o osso, e o tecido sinovial, independente do excesso de peso. ${ }^{14-16}$
Tais danificações, decorrentes da OA, afetam milhões de pessoas e apresentam como características principais a dor, a rigidez articular, e o declínio da funcionalidade, como, por exemplo, prejuízo na execução das atividades de vida diária (AVD).$^{17}$ As pesquisas destacam também que sua incidência aumenta com o avanço da idade, particularmente em indivíduos com mais de 60 anos. ${ }^{9,14-17}$

Visto as alterações na composição corporal durante o processo de envelhecimento e diante da importância da $\mathrm{OA}$ como fator de comprometimento funcional e da SM como fator de risco para doenças cardiovasculares, o presente estudo objetivou analisar a associação entre OA de joelho e SM em pacientes idosos não institucionalizados. $E$, considerando a escassez de estudos dessa natureza, principalmente com essa população, a presente pesquisa faz-se necessária inclusive para direcionar futuras políticas públicas na saúde do idoso.

\section{Metodologia}

Trata-se de uma pesquisa transversal, aleatorizada, extraída de um estudo probabilístico por conglomerado, intitulado "Análise Comparativa do Perfil Epidemiológico de Idosos de uma Comunidade: um Estudo de Coorte".

A amostra se deu por sorteio aleatório, através de cálculo amostral, de 416 indivíduos entre os 820 cadastrados na Unidade de Saúde da Família (USF) do Jardim Camanducaia/ Amparo, SP. Foram incluídos sujeitos nascidos até o ano 1948, residentes em Amparo, cadastrados nesta USF e que assinaram o Termo de Consentimento Livre e Esclarecido. Foram excluídos os idosos com deficiências físicas e/ou cognitivas severas (descritos nos registros médicos) e aqueles que não concluíram as etapas do estudo; $56.25 \%$ dos sujeitos não concluíram todas as etapas, logo a amostra final foi de 182 pacientes idosos.

Primeiramente aplicou-se de um questionário com dados sociodemográficos, antropométricos e condições de saúde. Posteriormente, realizou-se um raio-X de incidência anteroposterior de ambos os joelhos, e, por fim, foram colhidas amostras de sangue para análise laboratorial da glicemia de jejum e perfil lipídico - todos os indivíduos foram orientados a realizar jejum de 12 horas antes da coleta. 
312 Associação entre osteoartrite de joelho e síndrome metabólica Franco et al.

Tabela 1 Classificação dos idosos de acordo com gênero, idade e índice de massa corpórea

\begin{tabular}{|l|l|l|l|}
\hline Variáveis & $\mathbf{N}^{\mathbf{a}}$ & $\%$ & \multirow{2}{*}{ Média $\pm \mathbf{D P}^{\mathbf{b}}$} \\
\cline { 1 - 3 } Gênero & & \multicolumn{1}{|}{} \\
\cline { 1 - 3 } Feminino & 104 & 57,1 & \\
\cline { 1 - 3 } Masculino & 78 & 42,9 & \\
\cline { 1 - 3 } Total & 182 & 100,0 & $73,0 \pm 5,6$ \\
\hline Idade & 182 & & $27,9 \pm 4,8$ \\
\hline IMC $\left(\mathrm{kg} / \mathrm{cm}^{2}\right)$ & 182 & & \\
\hline
\end{tabular}

Abbreviation: DP, desvio padrão; IMC, índice de massa corpórea. ${ }^{a}$ Número de sujeitos.

${ }^{\text {b}}$ Desvio padrão.

O diagnóstico para OA considerou a escala de KellgrenLawrence $(\mathrm{KL} \geq 2){ }^{18}$ e para SM adotaram-se os critérios do NCEP, 4 o qual determina a presença de pelo menos 3 dos 5 fatores (ou uso de medicação): obesidade abdominal (circumferência da cintura [CC] $>102 \mathrm{~cm}$ e $88 \mathrm{~cm}$ para homens e mulheres respectivamente) ou central (índice de massa corpórea $\left.[\mathrm{IMC}]>30 \mathrm{Kg} / \mathrm{m}^{2}\right)$, hipertrigliceridemia $(>150 \mathrm{mg} / \mathrm{dL})$, baixos níveis de colesterol HDL ( $<35 \mathrm{mg} / \mathrm{dL}$ e $45 \mathrm{mg} / \mathrm{dL}$ para homens e mulheres respectivamente), hipertensão arterial sistêmica ( $>130 \times 85 \mathrm{mmHg}$ ) e glicemia de jejum elevada (> $100 \mathrm{mg} / \mathrm{dL}$.$) .$

O trabalho de campo foi realizado entre os anos de 2013 e 2014, por 3 pesquisadoras treinadas pela pesquisadora principal; o exame radiográfico foi realizado em uma clínica conveniada à prefeitura e os exames de sangue foram colhidos na USF e analisados pelo laboratório municipal.

Foi realizada uma análise exploratória de dados através de medidas resumo (média, desvio padrão, mínimo, mediana, máximo, frequência e porcentagem). A concordância entre os avaliadores foi verificada através do coeficiente Kappa. A comparação entre os grupos com e sem OA foi realizada através dos testes de Mann-Whitney ou Qui-quadrado, e a influência dos componentes da SM na OA foi avaliada através da regressão logística; no modelo múltiplo, o critério de variáveis usado foi o stepwise. $\mathrm{O}$ nível de significância adotado foi de $5 \%$.

O protocolo de pesquisa teve aprovação do Comitê de Ética em Pesquisa da nossa instituição sob o número $n^{\circ} 387.026$.

\section{Resultados}

A - Tabela 1 mostra uma maior proporção de idosos do sexo feminino (57,1\%), com média de idade de 73,0 $\pm 5,6$ anos e média de IMC de $27,9 \pm 4,8 \mathrm{~kg} / \mathrm{m}^{2}$.

A - Figura 1 apresenta a proporção de idosos diagnosticados ou não com SM e a - Figura 2 apresenta a proporção de idosos em relação ao número de componentes metabólicos acumulados na presença ou ausência da OA. Não houve associação significativa entre OA e o acúmulo de componentes $(p=0,6320)$, porém a maioria dos sujeitos com OA apresentou de 2 a 4 componentes.

A - Tabela 2 apresenta a associação da OA com idade, gênero e os componentes da $\mathrm{SM}$, sendo que a concordância entre os avaliadores para o diagnóstico da OA foi substancial para ambos os joelhos (coeficiente de Kappa direito = 0,7459 e esquerdo $=0,7527$ ). Foi encontrada relação da OA com CC (Valor de $p<0,0001$ ).

A - Tabela 3 apresenta a associação entre presença ou ausência de SM com a presença ou ausência de OA. Não houve associação significativa $(p>0,05)$.

A - Tabela 4 apresenta a influência da SM na OA. Verificou-se associação significativa da CC com a OA $(p<0,0001)$. $\mathrm{E}$, através de análise múltipla, evidenciou-se que o aumento de 1 centímetro na CC aumenta em 3,5 vezes a chance de OA $(\mathrm{OR}=3,524 ; \mathrm{IC} 95 \%=1,794-6,921)$.

\section{Discussão}

O presente estudo avaliou a associação entre OA de joelho e SM em pacientes idosos da comunidade, e os principais

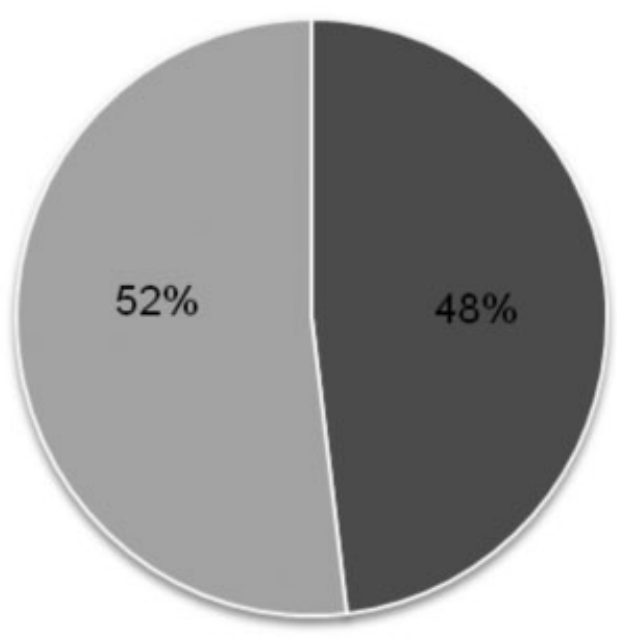

\section{m Ausência de SM $(<3$ componentes)}

\author{
Presença de SM ( $\geq 3$ \\ componentes)
}

\section{$\mathrm{SM}=$ Sindrome Metabólica}

Fig. 1 Proporção de idosos diagnosticados ou não com síndrome metabólica de acordo com o National Cholesterol Education Program Adult Treatment Panel III (ncep-atp iii). 


\section{Associação de OA de joelho com número de componentes metabólicos}

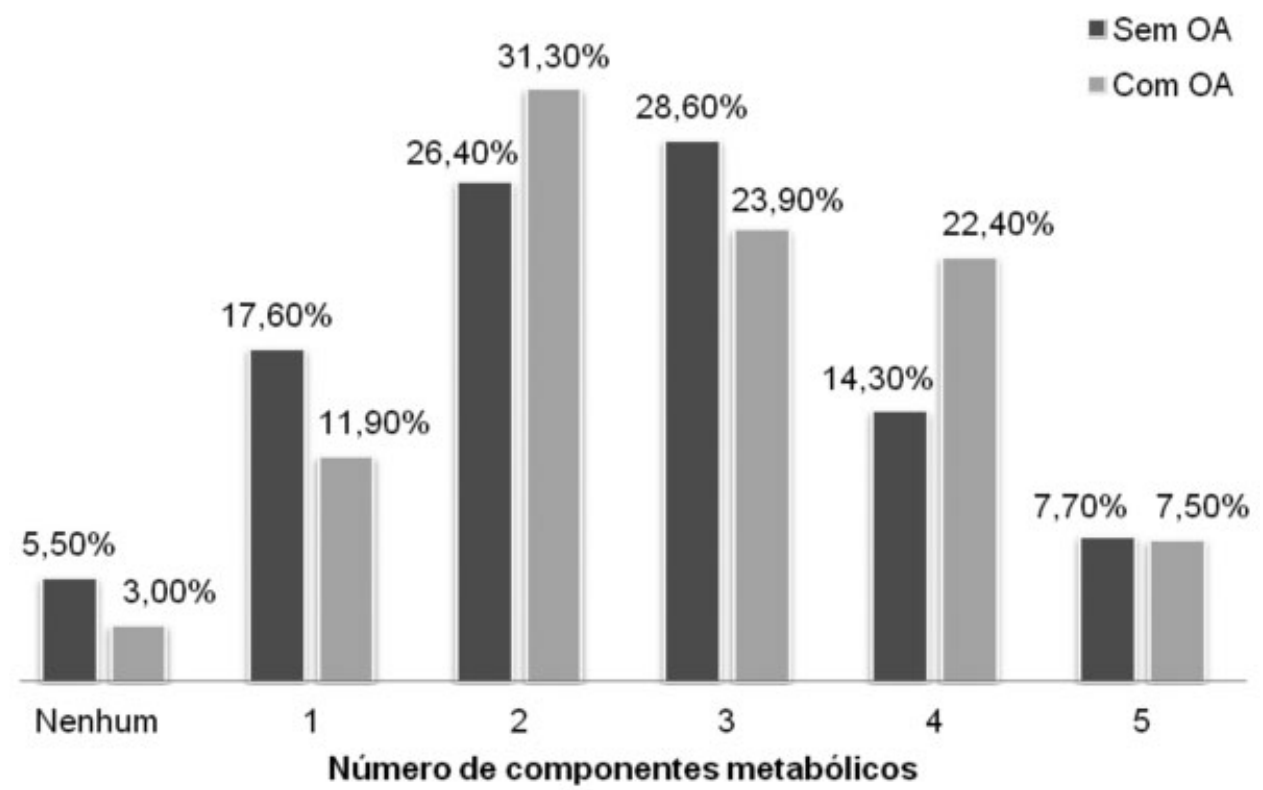

Fig. 2 Associação da osteoartrite de joelho com o número de componentes metabólicos em idosos.

resultados mostram associação da $\mathrm{OA}$ radiográfica de joelho com aumento da CC. Os sujeitos com OA apresentavam ainda as maiores idades, maiores níveis de IMC, maiores medidas de CC, maiores valores de hipertensão arterial sistêmica (HAS) e maiores níveis de triglicérides.

O primeiro grande estudo responsável por investigar essa associação (OA x SM) foi realizado em 2007, por Schett et al. ${ }^{16}$ Os pesquisadores acompanharam por mais de 20 anos 927 homens e mulheres com idade entre 40 e 80 anos. Os resultados mostraram taxas de artroplastia de joelho ou quadril decorrente de OA duas vezes maiores em pacientes com diabetes mellitus (DM) tipo 2, correlação entre o risco de artroplastia com a duração da diabetes e maiores níveis de inflamação sinovial e dor nos diabéticos. Esses dados reforçaram a hipótese da influência de componentes metabólicos na patogênese da $O A$.

Seguindo o mesmo caminho, Dahaghin et al. ${ }^{19}$ encontraram uma taxa duas vezes maior de OA de mão em pacientes diabéticos comparados com os não-diabéticos, evidenciando que a patogênese da OA independe de peso e sobrecarga.

Embora DM e OA não tenham apresentado associação estatisticamente significativa no presente estudo, outros autores observaram que a DM foi preditora de redução do espaço articular em homens com OA estabelecida de joelho ${ }^{20}$ e maior degradação articular em sujeitos diabéticos. ${ }^{21}$ Além disso, um modelo experimental ${ }^{22}$ demonstrou que ratos diabéticos apresentavam maior nível de glicose no sangue, diminuição das fibras de colágeno e proteoglicanos nos ligamentos e na cartilagem articular, e aumento do colágeno no tecido sinovial em comparação com ratos de controle, evidenciando a influência da DM na remodelação estrutural.

Em condições normais, o líquido sinovial humano contém baixas concentrações de colesterol comparadas aos níveis plasmáticos; ${ }^{23}$ no entanto, na presença de inflamação esses valores mostram-se elevados. Modelos animais ${ }^{24}$ têm mostrado associações entre dietas ricas em gordura com aumento da produção de citocinas pró-inflamatórias no líquido sinovial, formação de osteófitos e degradação da cartilagem articular, independente do peso corporal; e que a diminuição dos níveis de colesterol poderia minimizar esses efeitos.

Todavia, apesar de alguns autores relatarem uma ligação positiva entre hipercolesterolemia ${ }^{25-27}$ e OA, o presente estudo, assim como o de Eymard et al., ${ }^{20}$ mostraram uma correlação não significativa.

Em relação à HAS, pesquisas recentes ${ }^{13,25-27}$ apontam altos níveis de HAS nos sujeitos com OA, tal qual o estudo realizado por Jungmann et al. ${ }^{21}$ Neste estudo, os autores encontraram, em uma amostra de 1.000 pacientes com OA de quadril, uma taxa de prevalência de HAS e/ou doença cardiovascular (DCV) de 55\%.

Essa relação entre HAS e desgaste articular pode ser explicada partindo do ponto de a cartilagem ser um tecido avascular. Logo, o comprometimento no fluxo sanguíneo interfere negativamente nas trocas entre nutrientes e oxigênio ocasionando maior degeneração na cartilagem. ${ }^{11}$

Para Redon et al., ${ }^{12}$ a taxa de prevalência de HAS foi significativamente maior em pacientes com SM. O autor destacou também que em sujeitos hipertensos o risco de vir a desenvolver SM foi maior quando comparado com a população sem elevação pressórica. Em outro estudo com quase 1.400 hipertensos, ${ }^{13} 50 \%$ deles apresentaram comprometimento do metabolismo da glicose e SM associada, além de risco cardiovascular significativamente maior.

A presente pesquisa encontrou resultados de prevalência semelhantes à literatura, visto que no grupo com OA, 86,8\% dos idosos apresentaram HAS. Mas, apesar de essa associação 
314 Associação entre osteoartrite de joelho e síndrome metabólica Franco et al.

Tabela 2 Associação da osteoartrite de joelho com idade, gênero e os componentes metabólicos em idosos

\begin{tabular}{|c|c|c|c|c|}
\hline \multirow[b]{2}{*}{ Variável } & \multicolumn{4}{|c|}{ Osteoartrite de Joelho } \\
\hline & Sem OA & Com OA & Total & Valor de $p$ \\
\hline $\begin{array}{l}\text { Idade (Média } \pm \text { DP) } \\
\text { Total }\end{array}$ & $\begin{array}{l}72,3 \pm 5,6 \\
106\end{array}$ & $\begin{array}{l}73,9 \pm 5,5 \\
76\end{array}$ & $\begin{array}{l}73,0 \pm 5,6 \\
182\end{array}$ & $0,0571^{a}$ \\
\hline \multicolumn{4}{|l|}{ Gênero } & \multirow[t]{4}{*}{$0,6332^{b}$} \\
\hline Feminino & $59(55,7 \%)$ & $45(59,2 \%)$ & $104(57,1 \%)$ & \\
\hline Masculino & $47(44,3 \%)$ & $31(40,8 \%)$ & $78(42,9 \%)$ & \\
\hline Total & 106 & 76 & 182 & \\
\hline \multicolumn{4}{|c|}{ Circunferência da cintura } & \multirow[t]{4}{*}{$<0,0001^{b}$} \\
\hline Dentro do limite & $61(58,0 \%)$ & $21(27.0 \%)$ & $82(45,0 \%)$ & \\
\hline Fora do limite & $45(42,0 \%)$ & 55 (73.0\%) & $100(55,0 \%)$ & \\
\hline Total & 106 & 76 & 182 & \\
\hline \multicolumn{4}{|l|}{ Pressão Arterial } & \multirow[t]{4}{*}{$0,4808^{\mathrm{b}}$} \\
\hline Dentro do limite & $18(17,0 \%)$ & $10(13,2 \%)$ & $28(15,4 \%)$ & \\
\hline Fora do limite & $88(83,0 \%)$ & $66(86,8 \%)$ & $154(84,6 \%)$ & \\
\hline Total & 106 & 76 & 182 & \\
\hline \multicolumn{4}{|l|}{ Triglicérides } & \multirow[t]{4}{*}{$0,8274^{\mathrm{b}}$} \\
\hline Dentro do limite & $51(49,0 \%)$ & $39(50,7 \%)$ & $90(49,4 \%)$ & \\
\hline Fora do limite & $55(51,0 \%)$ & $37(49,3 \%)$ & $92(50,6 \%)$ & \\
\hline Total & 106 & 76 & 182 & \\
\hline \multicolumn{4}{|l|}{ HDL - colesterol } & \multirow[t]{4}{*}{$0,8881^{\mathrm{b}}$} \\
\hline Dentro do limite & $71(67,7 \%)$ & $51(66,7 \%)$ & $122(67,0 \%)$ & \\
\hline Fora do limite & $35(32,3 \%)$ & $25(33,3 \%)$ & $60(33,0 \%)$ & \\
\hline Total & 106 & 76 & 182 & \\
\hline \multicolumn{4}{|l|}{ Glicemia de jejum } & \multirow[t]{4}{*}{$0,0937^{\mathrm{b}}$} \\
\hline Dentro do limite & $61(57,5 \%)$ & $53(69,7 \%)$ & $114(62,6 \%)$ & \\
\hline Fora do limite & $45(42,5 \%)$ & $23(30,3 \%)$ & $68(37,4 \%)$ & \\
\hline Total & 106 & 76 & 182 & \\
\hline
\end{tabular}

Abreviaturas: DP, desvio padrão; HDL, high-density lipoprotein; N, número de sujeitos; OA, osteoartrite.

${ }^{\mathrm{a}}$ Teste de Mann-Whitney.

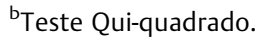

Tabela 3 Associação entre osteoartrite de joelho e síndrome metabólica em idosos

\begin{tabular}{|l|l|l|l|}
\hline \multicolumn{3}{|l|}{ Osteoartrite de joelho } \\
\hline Síndrome metabólica & Sem OA & Com OA & Valor de $p$ \\
\hline $\begin{array}{l}\text { Ausência SM } \\
(<3 \text { componentes) }\end{array}$ & $49,5 \%$ & $46,3 \%$ & $0,6924^{\text {a }}$ \\
\hline $\begin{array}{l}\text { Presença SM } \\
\text { ( } \geq 3 \text { componentes) }\end{array}$ & $50,5 \%$ & $53,7 \%$ & \\
\hline
\end{tabular}

Abreviaturas: OA, osteoartrite; SM, síndrome metabólica.

${ }^{a}$ Teste Qui-quadrado.

já ter sido relatada por alguns autores, ${ }^{21,28}$ HAS e OA não se associaram significativamente neste estudo. Yasuda et al., ${ }^{25}$ por sua vez, encontraram associação de HAS com a sintomatologia da $\mathrm{OA}$, porém não com sua gravidade e progressão radiológica.

A literatura também ressalta a associação da CC com OA. Maddah et al. $^{26}$ avaliaram a associação entre OA e SM e encontraram associação significativa entre CC e risco de OA. Para Eymard et al. ${ }^{20}$ e Shin, ${ }^{29}$ essa associação representou um aumento na gravidade dos sintomas, e, para Jungmann et al., ${ }^{21}$ Han et al. ${ }^{27}$ e Niu et al., ${ }^{28}$ ela traduziu um aumento na incidência de OA radiográfica. Assim como a referida literatura, a presente pesquisa encontrou associação entre aumento de CC e aumento de chance de OA.

Para Niu ${ }^{28}$ e Shin, ${ }^{29}$ o acúmulo de componentes metabólicos representou maior incidência de OA, mas, contrariando esses achados, o presente estudo não encontrou associação significativa entre eles.

Nesta pesquisa, curiosamente, dentre os idosos com OA, apenas 7,5\% apresentavam todos os 5 componentes da SM. Essa baixa prevalência de sujeitos com maior comprometimento metabólico pode ser explicada pela associação entre idade, multimorbidade e comprometimento funcional. ${ }^{30,31}$ 
Tabela 4 Razão de chances da influência dos componentes metabólicos na osteoartrite de joelho em idosos

\begin{tabular}{|l|l|l|l|}
\hline \multicolumn{4}{|l|}{ Análise simples (univariada) } \\
\hline Fator & RC & IC95\% (RC) & Valor de $p$ \\
\hline $\begin{array}{l}\text { Circunferência } \\
\text { da cintura }\end{array}$ & 3,729 & $\mathbf{1 , 9 4 9 ~ 7 , 1 3 3}$ & $<\mathbf{0 , 0 0 0 1}$ \\
\hline Pressão Arterial & 1,350 & $0,5853,116$ & 0,4819 \\
\hline Triglicérides & 0,935 & $0,5101 ., 714$ & 0,8275 \\
\hline HDL - colesterol & 1,048 & $0,5432,025$ & 0,8880 \\
\hline Glicemia jejum & 0,588 & $0,3161,097$ & 0,0950 \\
\hline $\begin{array}{l}\text { Análise múltipla } \\
\text { (multivariada) }\end{array}$ & & & \\
\hline Fator & $\mathrm{RC}$ & $\mathrm{IC95 \%}$ (RC) & Valor de $\boldsymbol{p}$ \\
\hline $\begin{array}{l}\text { Circunferência } \\
\text { da cintura }\end{array}$ & 3,524 & 1,794 6,921 & 0,0003 \\
\hline
\end{tabular}

Abreviaturas: HDL, high-density lipoprotein; IC, interval deconfiança; $R C$, razão de chances.

Esses fatores associados elevam a sintomatologia da OA e, neste caso, parecem ter dificultado o deslocamento dos participantes até os locais de coleta de exames. ${ }^{25,29}$

E, apesar de o envolvimento dos fatores metabólicos na etiologia da OA ser apoiado tanto por estudos epidemiológicos quanto por dados experimentais, alguns autores não encontraram associação significativa entre $\mathrm{OA}$ e $\mathrm{SM},{ }^{20,25}$ tal qual a presente pesquisa. Em contrapartida, Jungmann et al. $^{21}$ relataram que a OA aumentou a probabilidade de SM em mulheres, e Han et al. ${ }^{27}$ e Shin ${ }^{29}$ observaram que a SM aumentou a probabilidade de OA. Entretanto, essa correlação não se manteve significativa na maioria dos estudos após ajustes dos fatores confusão, $27-29$ tais como peso e IMC.

As discrepâncias nos resultados podem decorrer das diferenças amostrais - diferentes etnias e média de idade, e da falta de padronização dos critérios diagnósticos para OA e SM.

Dentre as limitações do estudo destacam-se: amostra composta por indivíduos de uma única região de um município, o que não implica em generalizações populacional; não consideração dos dados sociodemográficos; não consideração dos valores reais de cada componente da SM - sendo destacado apenas se o componente estava ou não alterado; deslocamento dos sujeitos - pode ter excluído sujeitos com maiores comprometimentos; e transversalidade do estudo impede a avaliação de relações diretas de causalidade entre as variáveis estudadas.

Embora não tenha sido encontrada relação entre SM e OA de joelho, são necessárias pesquisas semelhantes, principalmente longitudinais, em outros municípios brasileiros tanto com idosos da comunidade quanto com idosos institucionalizados, para que se possa acompanhar além da evolução das doenças, o nível de funcionalidade dos idosos e as implicações destas variáveis na saúde dos idosos. Investigações sobre as ações e influências das vias metabólicas na patogênese da OA também poderiam abrir uma nova avenida terapêutica e auxiliar na promoção de saúde desta população.

\section{Conclusão}

Conclui-se que apesar de a SM não se associar significativamente com OA de joelho, a medida da CC acima dos limites estabelecidos pela OMS está associada a maior chance de OA de joelho e aumenta a chance de SM em idosos não institucionalizados.

\section{Conflito de Interesses}

Os autores declaram não haver conflito de interesses.

\section{Agradecimentos}

Aos pesquisadores que contribuíram com a coleta e interpretação de dados e aos profissionais de saúde que participaram da pesquisa.

À Comissão de Aperfeiçoamento de Pessoal de Nível Superior (CAPES) [1741/2016; 3372/2017; 4588/2018], que nos deu apoio financeiro para realização deste trabalho.

\section{Referências}

1 Kluzek S, Newton JL, Arden NK. Is osteoarthritis a metabolic disorder? Br Med Bull 2015;115(01):111-121

2 Farnaghi S, Crawford R, Xiao Y, Prasadam I. Cholesterol metabolism in pathogenesis of osteoarthritis disease. Int J Rheum Dis 2017;20(02):131-140

3 World Health Organization. Definition, diagnosis and classification of diabetes mellitus and its complications: report of a WHO consultation. Part 1: Diagnosis and classification of diabetes mellitus (No. WHO/NCD/NCS/99.2). Geneva: World health organization; 1999

4 Expert Panel on Detection, Evaluation, and Treatment of High Blood Cholesterol in Adults. Executive Summary of The Third Report of The National Cholesterol Education Program (NCEP) Expert Panel on Detection, Evaluation, And Treatment of High Blood Cholesterol In Adults (Adult Treatment Panel III). JAMA 2001;285(19):2486-2497

5 Einhorn D, Reaven GM, Cobin RH, et al. American College of Endocrinology position statement on the insulin resistance syndrome. Endocr Pract 2003;9(03):237-252

6 Balkau B, Charles MA, Drivsholm T, et al. European Group For The Study Of Insulin Resistance (EGIR). Frequency of the WHO metabolic syndrome in European cohorts, and an alternative definition of an insulin resistance syndrome. Diabetes Metab 2002;28(05): 364-376

7 Associação Brasileira para o Estudo da Obesidade e da Síndrome Metabólica. Diretrizes brasileiras de obesidade 2009/2010. $3^{\mathrm{a}}$ ed. Itapevi,SP: AC Farmacêutica; 2009

8 Le Clanche S, Bonnefont-Rousselot D, Sari-Ali E, Rannou F, Borderie D. Inter-relations between osteoarthritis and metabolic syndrome: A common link? Biochimie 2016;121:238-252

9 Courties A, Sellam J, Berenbaum F. Metabolic syndrome-associated osteoarthritis. Curr Opin Rheumatol 2017;29(02):214-222

10 Grundy SM, Cleeman JI, Daniels SR, et al. Diagnosis and management of the metabolic syndrome: an American Heart Association/ National Heart, Lung, and Blood Institute Scientific Statement. Circulation 2005;112(17):2735-2752

11 Findlay DM. Vascular pathology and osteoarthritis. Rheumatology (Oxford) 2007;46(12):1763-1768

12 Redon J, Cifkova R, Laurent S, et al. Scientific Council of the European Society of Hypertension. The metabolic syndrome in hypertension: European society of hypertension position statement. J Hypertens 2008;26(10):1891-1900 
13 Carnevale Schianca GP, Fra GP, Steffanini M, et al. Impaired glucose metabolism in hypertensive patients with/without the metabolic syndrome. Eur J Intern Med 2014;25(05):477-481

14 Sellam J, Berenbaum F. Is osteoarthritis a metabolic disease? Joint Bone Spine 2013;80(06):568-573

15 Sun AR, Friis T, Sekar S, Crawford R, Xiao Y, Prasadam I. Is synovial macrophage activation the inflammatory link between obesity and osteoarthritis? Curr Rheumatol Rep 2016;18(09):57

16 Schett G, Kleyer A, Perricone C, et al. Diabetes is an independent predictor for severe osteoarthritis: results from a longitudinal cohort study. Diabetes Care 2013;36(02):403-409

17 Marques CDL, Duarte ALB. A importância do reconhecimento de comorbidades em pacientes com osteoartrite. Temas Reumatol 2011;12(01):3-6

18 Kellgren JH, Lawrence JS. The Epidemiology of Chronic Rheumatism: Atlas of Standard Radiographs of Arthritis. Oxford: Blackwell Scientific; 1963

19 Dahaghin S, Bierma-Zeinstra SM, Koes BW, Hazes JM, Pols HA. Do metabolic factors add to the effect of overweight on hand osteoarthritis? The Rotterdam Study. Ann Rheum Dis 2007;66(07):916-920

20 Eymard F, Parsons C, Edwards $\mathrm{MH}$, et al. Diabetes is a risk factor for knee osteoarthritis progression. Osteoarthritis Cartilage 2015;23 (06):851-859

21 Jungmann PM, Kraus MS, Alizai H, et al. Association of metabolic risk factors with cartilage degradation assessed by $\mathrm{T} 2$ relaxation time at the knee: data from the osteoarthritis initiative. Arthritis Care Res (Hoboken) 2013;65(12):1942-1950

22 Atayde SA, Yoshinari NH, Nascimento DP, et al. Experimental diabetes modulates collagen remodelling of joints in rats. Histol Histopathol 2012;27(11):1471-1479
23 Oliviero F, Lo Nigro A, Bernardi D, et al. A comparative study of serum and synovial fluid lipoprotein levels in patients with various arthritides. Clin Chim Acta 2012;413(1-2):303-307

24 Gierman LM, van der Ham F, Koudijs A, et al. Metabolic stressinduced inflammation plays a major role in the development of osteoarthritis in mice. Arthritis Rheum 2012;64(04): 1172-1181

25 Yasuda E, Nakamura R, Matsugi R, et al. Association between the severity of symptomatic knee osteoarthritis and cumulative metabolic factors. Aging Clin Exp Res 2018;30(05):481-488

26 Maddah S, Mahdizadeh J. Association of metabolic syndrome and its components with knee osteoarthritis. Acta Med Iran 2015;53 (12):743-748

27 Han CD, Yang IH, Lee WS, Park YJ, Park KK. Correlation between metabolic syndrome and knee osteoarthritis: data from the Korean National Health and Nutrition Examination Survey (KNHANES). BMC Public Health 2013;13:603

28 Niu J, Clancy M, Aliabadi P, Vasan R, Felson DT. Metabolic syndrome, its components, and knee osteoarthritis: the Framingham osteoarthritis study. Arthritis Rheumatol 2017;69(06): 1194-1203

29 Shin D. Association between metabolic syndrome, radiographic knee osteoarthritis, and intensity of knee pain: results of a national survey. J Clin Endocrinol Metab 2014;99(09):3177-3183

30 Chi WC, Wolff J, Greer R, Dy S. Multimorbidity and decisionmaking preferences among older adults. Ann Fam Med 2017;15 (06):546-551

31 Yarnall AJ, Sayer AA, Clegg A, Rockwood K, Parker S, Hindle JV. New horizons in multimorbidity in older adults. Age Ageing 2017; 46(06):882-888 